\title{
O futebol recreativo como uma nova abordagem terapêutica para a obesidade em crianças e adolescentes: uma revisão sistemática
}

\author{
Recreational soccer as a novel therapeutic approach to children and \\ adolescent obesity: a systematic review
}

Augusto Pedrett', Alessandro Pedretti', Fabrício Vasconcellos², André Seabra ${ }^{3}$

\section{Resumo}

O aumento da prevalência de sobrepeso e obesidade tem se tornado um grave problema de saúde pública. No Brasil dados recentes sugerem que o sobrepeso atinge mais de $30 \%$ das crianças entre os cinco e os nove anos de idade e cerca de $20 \%$ da população entre os 10 e os 19 anos. A atividade física é considerada uma importante ferramenta para a prevenção e tratamento da obesidade infantil. O futebol, um dos desportos mais populares no mundo, tem sido considerado uma abordagem importante e eficaz para a promoção da saúde através da atividade física. O objetivo do presente estudo foi revisar o estado atual do conhecimento sobre a influência de programas de futebol recreativo (PFR) na prevenção e tratamento da obesidade e suas comorbidades associadas em crianças e adolescentes. Uma extensa pesquisa em bases de dados eletrônicas online foi realizada na Medline, ScienceDirect, LILACS, Scholar Google, Scopus e SPORTDiscus. Nove estudos preencheram todos os critérios de inclusão, com um total de 214 participantes que completaram seus respectivos programas de futebol recreativo. Embora um pequeno número de estudos tenha sido encontrado, os resultados reportados sugerem que a participação nos PFR pode melhorar a composição corporal, fatores de risco metabólicos e variáveis do bem-estar psicológico de crianças com sobrepeso e obesidade. Encontramos evidências de que a prática do futebol recreativo pode representar uma opção eficaz e acessível para a prevenção e tratamento da obesidade e suas comorbilidades associadas em crianças e adolescentes.

\section{Abstract}

The raising level of overweight and obesity prevalence has become a serious public health problem. Recent Brazilian data suggest that overweight accounts for $30 \%$ of all children from five to nine years old and around 20\% of the 10 to 19 population. Physical activity is considered an important tool for the prevention and treatment of child obesity. And soccer, one of the most popular sports in the world, has being considered and important and effective approach to promote health through physical activity, being yet of easy implementation and low investment. The objective of the present study was to review the current state of knowledge about the influence of recreational soccer programs (RSP) in the prevention and treatment of child and adolescent obesity and its associated comorbidities. An extensive search of online electronic databases was held in Medline, ScienceDirect, LILACS, Google Scholar, Scopus, and SPORTDiscus. Nine studies met all inclusion criteria for this review, with a total of 214 participants completing their respective recreational soccer program. Despite the low number of studies found, the reported results suggest that RSP's can improve the body composition profile, metabolic risk factors and psychological well-being variables of overweight and obese children. We found evidences that the practice of recreational soccer may represent an effective and available option to the prevention and treatment of obesity and it associated comorbidities in children and adolescents.

\section{Keywords}

Overweight; Children; Intervention.

\section{Palavras-chave}

Sobrepeso; Criança; Intervenção.

1 Centro de Investigação, Formação, Intervenção e Inovação em Desporto (CIFI2D) Faculdade de Desporto, Universidade do Porto, Portugal.

2 Laboratório de Atividade Física e Promoção da Saúde, Universidade Estadual do Rio de Janeiro, Brasil.

3 Centro de Investigação em Actividade Física, Saúde e Lazer (CIAFEL), Faculdade de Desporto, Universidade do Porto, Portugal.

\section{Introdução}

Durante as últimas décadas tem se observado um incremento muito significativo na prevalência da obesidade em crianças e adolescentes ${ }^{1-3}$, contribuindo para que a obesidade seja já considerada uma epidemia mundial. Este aumento que se tem vindo a verificar é 
extraordinariamente alarmante, pois a obesidade traz associadas diversas comorbidades de que se destacam a diabetes, a hipertensão e o desenvolvimento de fatores de risco de doenças cardiovasculares ${ }^{4,5}$. $\mathrm{Na}$ literatura estão bem identificadas às causas para todas as comorbidades que lhe estão associadas, estando o sedentarismo como um dos fatores que mais contribui para este quadro alarmante $e^{5,6}$.

A Pesquisa de Orçamento Familiar realizada pelo Instituto Brasileiro de Geografia e Estatística ${ }^{7}$ mostra que no Brasil, em todas as faixas etárias e estratos socioeconômicos, é cada vez mais elevado o percentual de crianças e adolescentes com excesso de peso e obesidade. $\mathrm{Na}$ atualidade, o excesso de peso atinge mais de $30 \%$ das crianças entre os cinco e os nove anos de idade e cerca de $20 \%$ da população entre 10 e 19 anos de idade. Aquelas entre cinco e 19 anos com renda maior que cinco salários mínimos apresentam prevalência de excesso de peso e obesidade se comparado aquelas com renda até $1 / 4$ do salário mínimo .

Problemas consideráveis para os sistemas de saúde pública ${ }^{2,3,8}$ do ponto de vista da sociedade manifestam-se em dois principais tipos de custo econômico - os custos diretos, relacionados com as despesas do sistema de saúde; dos pacientes e dos seus familiares com o tratamento, prevenção e diagnóstico; e ainda despesas com reabilitação, investigação, formação e investimento em saúde. Outro tipo de custos econômicos reporta-se aos custos indiretos, que representam o valor da produção perdida devido à doença e à morte prematura, podendo ainda compreender, adicionalmente, o tempo de lazer e de trabalho perdido, sacrificado pelos familiares e amigos para acompanharem os doentes?.

Face ao quadro exposto parece-nos claro que a obesidade e as comorbidades que lhe estão associadas merecem prevenção urgente. A atividade física (AF) é considerada uma estratégia importante para essa prevenção ${ }^{10,11}$, tendo diversas pesquisas investigado a eficácia de programas de AF no "combate" à obesidade infantil ${ }^{11-13}$. Embora limitados e não consensuais, os resultados encontrados parecem sugerir um efeito positivo da participação regular em programas de AF na prevenção da obesidade.

O futebol recreativo tem sido recentemente investigado e se destacado como eficaz na promoção de saúde de crianças e adolescentes com sobrepeso e obesida$\mathrm{de}^{14,15}$, no qual requer uma enorme participação do componente aeróbio e um elevado gasto energético ${ }^{16,17}$, além de envolver atividades de alto impacto que estimulam o sistema musculoesquelético conforme as recomendações da Organização Mundial da Saúde ${ }^{18}$ a fim de melhorar o condicionamento cardiorrespiratório, o sistema musculoesquelético, os biomarcadores cardiovasculares e metabólicos e a redução da ansiedade e depressão.

De tal modo, acreditamos que por o futebol ser um esporte altamente popular, culturalmente e socialmente importante e acessível a todos os estratos sociais, poderá ser uma importante estratégia para a prevenção da obesidade em crianças e adolescentes. O presente estudo tem como principal objetivo revisar o estado atual do conhecimento sobre a influência de programas de futebol recreativo para a prevenção da obesidade em crianças e adolescentes e das comorbidades que the estão associadas.

\section{Métodos}

Uma extensa pesquisa de bases de dados eletrônicas online foi realizada na Medline, ScienceDirect, LILACS, Scholar Google, Scopus, e SPORTDiscus nos últimos 15 anos. As palavras do texto, palavras-chave e descritores utilizados nas pesquisas foram: (children OR adolescent) AND (prevent OR intervention) AND (physical activity) AND 
(obesity OR overweight) AND (increase OR gain OR change) AND (BMI OR "body mass index" OR "fat mass") AND (soccer OR football). Os critérios de inclusão foram estudos publicados em Inglês, com crianças e adolescentes com sobrepeso e obesidade, definido a partir de percentis do índice de massa corporal (IMC) para sexo e idade-específica. O IMC é um dos indicadores mais utilizados para determinar o estado nutricional de crianças e adolescentes, sendo definidas com sobrepeso, todas aquelas que para sexo e idade-específica estão entre o percentil 85 e 95 do seu IMC, ao passo que obesidade é definida para um IMC superior ao percentil $95^{7}$. Estudos foram elegíveis para inclusão na presente revisão se tivessem aplicado o futebol recreativo como programa de intervenção (atividade física), isolada ou combinada com outro tipo de intervenção (por exemplo, estilo de vida ou dieta), independentemente da sua duração. Entende-se por futebol recreativo, o futebol ao alcance comum, praticado de forma lúdica e sem regras rígidas, equipamentos e uniformes especiais, ou qualquer outra formalidade e disciplina de jogo, conhecido popularmente como "pelada", possui, no mundo todo e em especial no Brasil, uma "dimensão de massa". Foram excluídas as publicações repetidas para os mesmos estudos. Na presença de qualquer dúvida sobre a inclusão do estudo, uma decisão final foi dada após o texto completo ter sido analisado. As referências de todos os estudos previamente incluídos foram examinadas para detectar outros possíveis estudos relevantes não identificados pela pesquisa de banco de dados.

\section{Resultados}

Identificaram-se pela busca eletrônica 528 estudos potencialmente relevantes e pela busca manual das listas de referência identificaram-se outros cinco. Um total de nove estudos preencheram todos os critérios de inclusão para esta revisão. Um total de 214 participantes entre oito e 17 anos foi submetido a programas de futebol recreativo e completaram seus respectivos estudos. Em todos os estudos, um nível de significância de 0,05 foi estabelecido para o erro tipo I. A Figura 1 resume o processo de seleção dos estudos incluídos.

O Quado 1 apresenta as características dos nove estudos incluídos na revisão sistemática. Na totalidade dos estudos elegidos (exceto Vasconcellos e colegas que buscam estratégias de tratamento) o principal "objetivo" foi o de contribuir com estratégias para a prevenção das comorbidades associadas à obesidade em crianças com excesso de peso.

Seis dos nove estudos propuseram o futebol recreativo como única intervenção ${ }^{14,19-23}$ e quatro estudos incluíram para além do PFR outro tipo de intervenção (estilo de vida ou reeducação alimentar) ${ }^{15,24-26}$. Seis estudos incluíram meninos e meninas $^{14,15,21,23,25,26}$, um incluiu somente meninos ${ }^{25}$ enquanto dois incluíram somente meninas ${ }^{15,24}$. Três estudos tiveram programas de intervenção não superior a 12 semanas ${ }^{21,23,24}$, enquanto sete estudos forneceram acompanhamento mais longo $^{14,15,19,20,22,25,26}$.

O projeto-piloto ${ }^{22}$ envolvendo crianças/adolescentes com idades compreendidas entre oito e 13 anos, a prática do futebol recreativo por 50 minutos três vezes por semana durante seis meses resultou na melhoria de marcadores somáticos associados à saúde, ou seja, na redução significativa do zsc-IMC, da gordura corporal total e da gordura intra-abdominal (DXA), bem como da razão cintura/ altura. Resultou também no aumento da massa muscular, do conteúdo mineral ósseo e da densidade mineral óssea (DXA). Efetivamente, e no que respeita aos marcadores de risco cardiometabólicos, observou-se uma redução significativa do colesterol total, do colesterol das LDL e dos triglicerídeos, bem como da pressão 


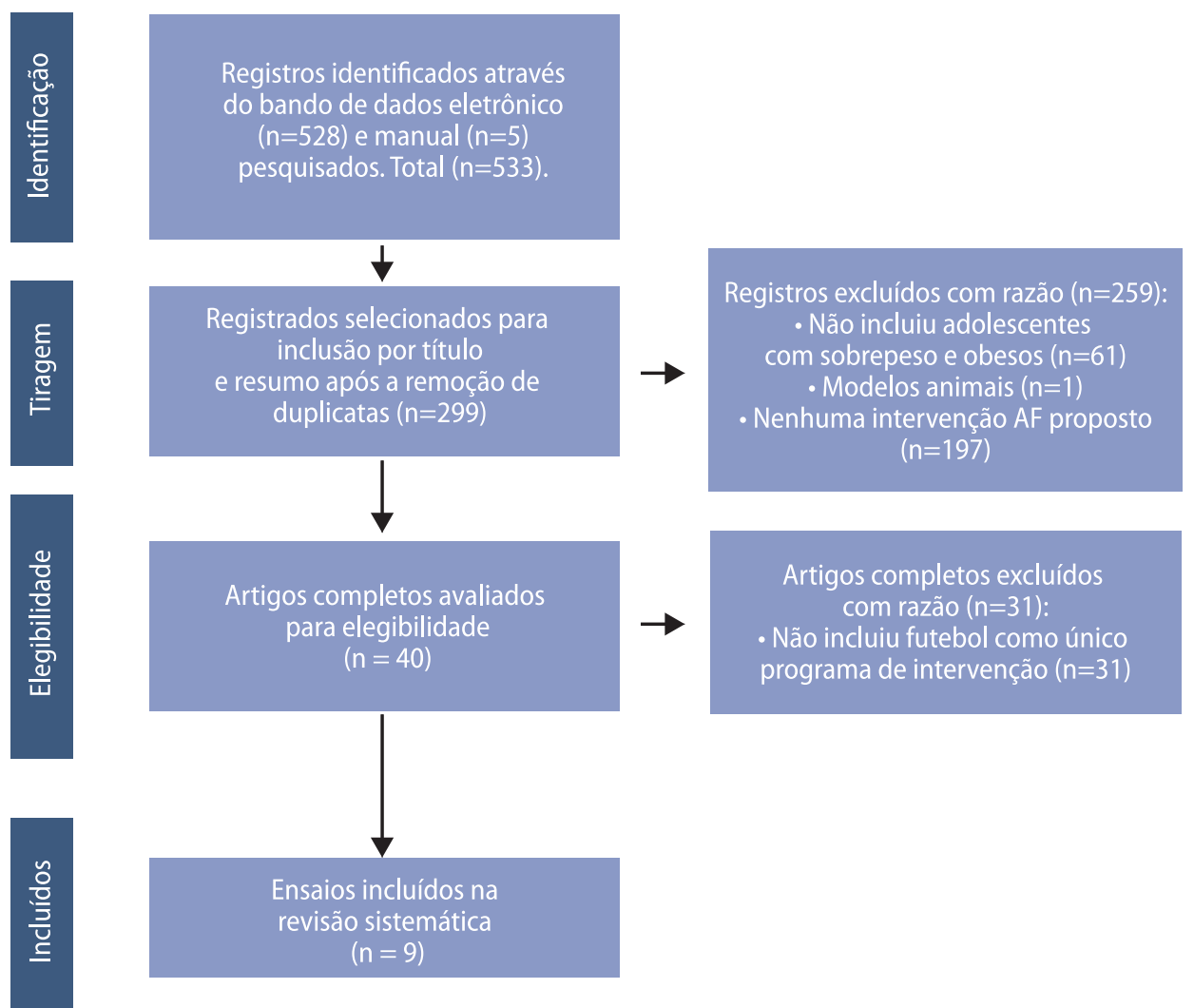

FIGURA 1 - Fluxograma ilustrando os detalhes da estratégia de busca, triagem dos resultados potencialmente qualificados (n), a seleção dos estudos incluídos e as razões para a exclusão dos mesmos. AF: atividade física

arterial diastólica e um aumento do colesterol das HDL. Registrou-se ainda redução significativa dos valores da leptina, da resistina e da fração oxidada das LDL e aumento da adiponectina.

Faude e colaboradores ${ }^{14}$ com o objetivo de analisar a eficácia de um PFR ( $n=11$; cinco meninas) durante seis meses, três vezes por semana, em comparação com um programa de treino tradicional (PTT; $n=11$; três meninas), avaliou meninos e meninas obesas da Alemanha, de oito a 12 anos, em suas características antropométricas (altura e peso corporal). Seu estudo não evidenciou diferença significativa inter e intra grupos para a antropometria. Seabra et al. ${ }^{25}$ evidenciaram alguns resultados semelhantes ao analisar a eficácia de um PFR $(n=12)$ durante cinco meses, quatro vezes por semana, em comparação com um grupo controle (GC; $n=8)$, em crianças portuguesas com idade entre oito e 12 anos. As crianças em ambos os grupos exibiram um aumento significativo na estatura $(p<0,001)$. No entanto, o ganho em estatura foi mais pronunciado no PFR $(p=0,03)$. Ambos os grupos apresentaram aumentos significativos no peso corporal e massa corporal magra $(p<0,05)$, enquanto não foram observadas alterações significativas para o IMC e percentual de gordura corporal após cinco meses de intervenção $(p>0,05)$.

Indo de encontro a esses resultados, Tabor ${ }^{20}$ encontrou que o PFR teve um efeito não significativo na classificação do peso corporal ao final do estudo. Já Hansen e colegas ${ }^{21}$, para ambos os grupos (PFR e CG), mostrou que o peso corporal manteve-se estável durante o período de três meses, e ambos aumentaram comparativamente a estatura. No entanto, Weintraub et. al. ${ }^{15}$ observaram efeitos benéficos sobre o IMC, IMC ' $z$ escore', no total de atividade física diária e no total de minutos dispendidos em atividade física moderado-vigorosa (AFMV) aos três 
QUADRO 1 - Características dos estudos que examinaram o efeito da atividade física sobre o excesso de peso e obesidade na adolescência.

\begin{tabular}{|c|c|c|c|c|c|c|c|c|}
\hline Estudo & $\begin{array}{c}\text { Estado de } \\
\text { obesidade [IMC } \\
-\mathrm{kg} / \mathrm{m}^{2} \text {, percentil] }\end{array}$ & Ano & $\begin{array}{c}\text { Tamanho } \\
\text { da amostra } \\
\text { (intervenção) }\end{array}$ & País & Sexo & $\begin{array}{l}\text { Idade, anos } \\
\text { (intervalo ou } \\
\text { média) }\end{array}$ & $\begin{array}{c}\text { Atividade Física } \\
\text { (semanal em minutos) }\end{array}$ & $\begin{array}{l}\text { Duração } \\
\text { (semanas) }\end{array}$ \\
\hline $\begin{array}{l}\text { Faude } \\
\text { et al. }{ }^{14}\end{array}$ & $\geq 95$ & 2010 & $\begin{array}{l}\mathrm{PFR}=11 \\
\mathrm{PTT}=11\end{array}$ & Alemanha & $M / F$ & $8-12$ & $3 x / 5-$ Futebol $-60 \mathrm{~min}$ & 24 \\
\hline Tabor $^{20}$ & $\geq 85^{* *}$ & 2014 & PFR $=62$ & Estados Unidos & $M / F$ & $8-10$ & CLOCC & 96 \\
\hline $\begin{array}{l}\text { Hansen } \\
\text { et al. }{ }^{21}\end{array}$ & $\geq 85$ & 2013 & $\begin{array}{l}\mathrm{PFR}=20 \\
\mathrm{GC}=11\end{array}$ & Portugal & $M / F$ & $8-12$ & $\begin{array}{c}4 x / S-\text { Futebol }-60- \\
90 \mathrm{~min}\end{array}$ & 12 \\
\hline Leach e Yates ${ }^{24}$ & $\geq 95$ & 2008 & $\begin{aligned} \mathrm{PFR} & =11 \\
\mathrm{GC} & =7\end{aligned}$ & Estados Unidos & $\mathrm{F}$ & $10-11$ & FITNESSGRAM & 8 \\
\hline $\begin{array}{l}\text { Madsen } \\
\text { et al. }{ }^{19}\end{array}$ & $\geq 85^{*}$ & 2013 & $\begin{array}{l}\mathrm{PFR}=79 \\
\mathrm{GC}=71\end{array}$ & Estados Unidos & $M / F$ & $9-11$ & $\begin{array}{c}2-3 x / 5-\text { Futebol }- \\
120 \mathrm{~min}\end{array}$ & 40 \\
\hline $\begin{array}{l}\text { Seabra } \\
\text { et al. }{ }^{25}\end{array}$ & $\geq 85$ & 2014 & $\begin{aligned} \mathrm{PFR} & =12 \\
\mathrm{GC} & =8\end{aligned}$ & Portugal & M & $8-12$ & $\begin{array}{c}4 \mathrm{x} / \mathrm{S}-\text { Futebol }-60- \\
90 \mathrm{~min}\end{array}$ & 20 \\
\hline Weintraub et al. ${ }^{15}$ & $\geq 85$ & 2008 & $\begin{array}{l}\mathrm{PFR}=9 \\
\mathrm{GC}=12\end{array}$ & Estados Unidos & $\mathrm{F}$ & $9-11$ & $3-4 x / S-$ Futebol $-75 \mathrm{~min}$ & 24 \\
\hline Rêgo e Seabra ${ }^{22}$ & $\geq 85$ & 2014 & PFR & Portugal & - & $8-13$ & $3 x / 5-$ Futebol $-50 \mathrm{~min}$ & 24 \\
\hline Vasconcellos et al. ${ }^{23}$ & $\geq 85$ & 2015 & $\begin{array}{l}\mathrm{PFR}=10 \\
\mathrm{GC}=10\end{array}$ & Brasil & $M / F$ & $12-17$ & $3 x / 5-$ Futebol $-60 \mathrm{~min}$ & 12 \\
\hline
\end{tabular}

PFR: programa de futebol recreativo; PTT: programa de treino tradicional; GC: grupo controle; representado por *40\% e **50\% da amostra.

e seis meses de intervenção. Assim como todas as crianças alocadas ao PFR ( $n=9)$ e $42 \%$ das crianças que participaram do programa de educação para a saúde (PES; $n=12$ ) apresentaram menores IMC ' $z$ escores' nos três e seis meses de intervenção ao avaliar a viabilidade, aceitabilidade e eficácia para reduzir o ganho de peso em crianças com excesso de peso de baixa renda (9-11 anos de idade) de meninas americanas. Houve também interação significativa entre os valores basais do IMC ' $z$ escore' e a intervenção aos três e seis meses $(p=0,04)$.

Ao final de 10 meses, meninos e meninas obesas dos EUA, de 9 a 11 anos, tiveram avaliados sua alteração no AFMV após a escola e mudanças no IMC e IMC ' $z$ escores' por Madsen et. al. ${ }^{19}$, com o objetivo de analisar a eficácia de um PFR ( $n=79$; 38 meninas), quatro vezes por semana, em comparação com um GC ( $n=71 ; 42$ meninas). Os alunos do PFR acumularam mais tempo de AFMV após a escola do que os do GC, sem outras diferenças entre os grupos. No geral, os alunos realizaram mais AFMV em dias úteis do que aos sábados e o acúmulo aumentou significativamente 3,4 minutos após a escola e 18,5 minutos aos sábados, apesar de não haver diferenças no IMC ' $z$ escores'. Já as análises de Leach e Yates ${ }^{24}$ revelaram que o IMC aumentou ligeiramente em ambas as condições, experimentais e de controle, não revelando diferenças significativas entre os grupos $(p=0,80)$. No entanto, as estatísticas descritivas revelaram assimetria significativa e curtose dos dados pré e pós-mudança do IMC dentro dos participantes aleatorizados no PFR. Meninas americanas de 10 e 11 anos, com intuito de reunir evidências preliminares sobre possíveis benefícios do futebol recreativo e educação nutricional, foram inseridas no PFR $(n=11)$ durante dois meses e comparadas a um GC $(n=7)$, após cinco meses de educação nutricional.

No Brasil ${ }^{23}, 20$ adolescentes com idades entre 12-17 anos foram divididos em dois grupos, o grupo do PFR ( $n=10$; duas meninas) e o grupo controle ( $n=10$; quatro meninas). O programa de futebol recreativo foi ministrado durante 12 semanas, três vezes por semana, com 60 minutos de atividades por sessão. Ao final da intervenção, o PFR exibiu reduções significativas no IMC, circunferência da cintura, percentual de gordura corporal, diminuição da pressão arterial sistólica, do 
colesterol total, dos trigliceres, proteína C-reativa, resistência à insulina e da atividade simpática se comparado ao grupo controle.

Hansen e colegas ${ }^{21}$ examinaram os efeitos de um PFR de três meses em crianças com sobrepeso por meio de ecocardiografia e tonometria arterial periférica. Meninos e meninas obesas de Portugal com 10 e 11 anos tiveram avaliadas sua estrutura e função cardíaca e função arterial periférica com o objetivo de se analisar a eficácia do PFR ( $n=20$; três meninas) durante três meses, três vezes por semana, em comparação com um GC ( $n=11$; quatro meninas). Após três meses, a pressão arterial sistólica aumentou significativamente no GC ( $p<0,05 ; 112$ vs. 122 $\mathrm{mmHg}$ ), mas diminuiu no PFR ( $p=0,05 ; 114$ vs. $112 \mathrm{mmHg}$ ), observando diferença significativa entre os grupos ao final dos três meses $(p<0,05)$, enquanto a pressão arterial diastólica não se alterou significativamente em ambos os grupos durante o período de estudo. Nenhuma mudança significativa foi observada na frequência cardíaca de repouso após três meses. No estudo de Faude e colaboradores ${ }^{14}$ também foi avaliado a aptidão física em bicicleta ergométrica. No teste ergométrico, a potência máxima aumentou significativamente para ambos os grupos (PFR e PTT) durante os três primeiros meses $(p<0,01), o \mathrm{VO}_{2 \max }$ e a concentração de lactato mantiveram-se constante ao longo dos meses $(p>0,05)$. A FC $\mathrm{max}_{\max }$ aumentou significativamente $(p=0,02)$ nos três meses finais, enquanto a FC submáxima diminuiu significativamente durante os três primeiros meses $(p<0,01)$ e nos três meses seguintes $(p<0,01)$. Não houve diferença significativa inter grupo para nenhuma das variáveis analisadas $(p>0,05)$. Por outro lado, Madsen et. al. ${ }^{19}$ não evidenciou diferenças significativas na aptidão física em seu estudo.

No início do estudo de Hansen e colegas ${ }^{21}$, todos os parâmetros foram similares nos dois grupos (PFR e GC). Após três meses, o diâmetro da parede posterior do ventrículo esquerdo aumentou significativamente no PFR $(p<0,001)$, mas não no GC, enquanto o diâmetro do septo interventricular não se alterou significativamente. O diâmetro diastólico do ventrículo direito diminuiu significativamente após três meses no GC $(p=0,03)$, com semelhante diminuição ainda que não significativa foi observado no PFR $(p=0,07)$. Após três meses, houve um aumento substancial da função sistólica do ventrículo direito no $\operatorname{PFR}(p<0,01)$, mas não no GC, e observou-se aumento do tempo global de relaxamento isovolumétrico após três meses no PFR ( $p<0,001)$, enquanto nenhuma alteração foi observada em GC. Para além dos benefícios já considerados ${ }^{22,23}$, salienta-se igualmente a melhoria significativa da aptidão cardiorrespiratória e, consequentemente, a maior aptidão para realizar exercício prolongado com menor fadiga ${ }^{22}$, assim como o aumento significativo da atividade parassimpática, $\mathrm{VO} 2_{\text {pico }}$ e colesterol HDL e diminuição da resistência vascular no PFR, mas não nos controles ${ }^{23}$.

Faude e colaboradores ${ }^{14}$ no estudo já apresentado também procurou analisar a saúde subjetiva, bem como a habilidade motora em meninos e meninas alemãs. Não foram observadas diferenças significativas entre os grupos (PFR e PTT) para o total, bem como para todas as seis subescalas do Questionário de Qualidade de vida para Crianças (KINDL-R) que analisou: bem-estar físico; bem-estar emocional; auto-estima; família; amigos; escola. O principal efeito significativo ao longo da intervenção só foi evidenciada para a subescala de auto-estima, com um aumento ao longo do tempo e um efeito maior no PFR. Sendo o mesmo observado no estudo de Seabra et al..$^{25}$, em que os participantes do PFR também melhoraram sua auto-estima $(p<0,05)$. Por outro lado, Weintraub et. al. ${ }^{15}$ mostrou não haver diferença evidente entre os grupos (PFR e GC) quanto a auto-estima. Foi observada por Faude e colaboradores ${ }^{14}$ uma interação significativa para a subescala escola 
( $p=.03$ ), ocorrendo um aumento de $10 \%$ no PFR, e uma diminuição de $15 \%$ para o PTT. Não foram observadas diferenças significativas entre os grupos para o questionário KINDL-R ( $p>0,05)$. Quanto às capacidades motoras, Faude e colaboradores ${ }^{14}$ evidenciaram melhoras significativas nas sete capacidades analisadas durante o período de intervenção: Countermovement jump $(p<0,01)$; Sit-and-reach test $(p<.001)$; One-leg-standing ( $p<0,001)$; Agility run ( $p<0,001) ; 20$ m-shuttle-run test $(p<0,001)$.

Seabra et $a l .{ }^{25}$ analisaram também indicadores do estado psicológico percebido, incluindo imagem corporal, autoestima, atração em participar em atividade física e competência física percebida através de questionários padronizados nas crianças portuguesas. Ao longo dos cinco meses de intervenção as maiores mudanças significativas foram observadas no estado psicológico percebido do PFR em comparação com o GC. Os participantes do PFR também melhoraram sua imagem corporal, e se perceberam como mais bem sucedido e competente fisicamente do que o GC. Assim como os meninos no PFR mostraram maior tendência em participar de AF com uma significativa interação, sugerindo que o PFR aumentou a propensão para participação em AF, enquanto o GC permaneceu constante em sua simpatia por AF ao longo de todo o período de intervenção. Em seu projeto-piloto ${ }^{22}$, Rêgo e Seabra também registram na vertente comportamental mudanças significativas nas variáveis de auto percepção psicológica. A melhoria da imagem corporal, da autoestima, da qualidade de vida e da percepção de sucesso pessoal e competência física, assim como na atitude de maior motivação intrínseca para a participação em AF.

Outras evidências foram relacionadas pelos estudos elegidos. Leach e Yates ${ }^{24}$ realizaram cinco meses de reeducação alimentar antes de iniciar o PFR. A reeducação alimentar de 15 minutos aumentou significativamente o conhecimento dos alunos sobre o "USDATips for Families resource" e os indivíduos que completaram a pesquisa mantiveram esse conhecimento ao longo do período de cinco meses. No entanto, não se correlacionou com a diminuição do IMC $(p=0,48)$. Outra informação relevante que Madsen et. al. ${ }^{19}$ mostrou em seu estudo é que a participação geral no programa de intervenção foi de $48 \%$, com os alunos com sobrepeso e obesos mais assíduos do que os estudantes com peso normal (60\% vs. 39\%). Já Tabor $^{20}$ evidenciou que as crianças que optaram pelo desporto de equipe eram mais propensas a ser obesos do que as que optaram pelo esporte individual e que os meninos são mais propensos a serem obesos que as meninas.

Tabor $^{20}$ analisou a redução da obesidade e o aumento do desempenho acadêmico em crianças de baixa renda por meio da análise a respeito da participação esportiva, desempenho acadêmico e o comportamento em sala de aula e o IMC durante 24 meses em meninos e meninas dos EUA, entre oito e 10 anos, que participaram de um PFR $(n=62)$. Não houve efeito significativo do PFR no desempenho acadêmico ao final dos 24 meses, assim como nenhuma das interações foram significativas entre gênero ou etnia e o PFR sobre o desempenho acadêmico. Além disso, a capacidade dos alunos se concentrarem, aprenderem e sua ética não foi significativamente relacionada com o desempenho acadêmico. No entanto, a capacidade dos alunos para terminar o que eles ou elas começaram foi significativamente relacionada com o desempenho acadêmico $(p<0,01)$, tanto na matemática quanto para a leitura $(p<0,01)$.

Quanto à "preocupação com sobrepeso", Weintraub et. al. ${ }^{15}$ mostrou não haver diferença evidente entre os grupos. Ao final dos seis meses, $89 \%$ das crianças que compunham o PFR afirmaram que gostariam de continuar jogando em uma equipe de futebol. 


\section{Discussão}

Este estudo objetivou revisar o estado atual do conhecimento sobre a influência de programas de futebol recreativo para a prevenção da obesidade e comorbidades associadas em crianças e adolescentes. As variáveis relacionadas à prática de futebol recreativo foram de caráter somático, bioquímico, inflamatório, cardiovascular e psicológico.

Os PFR evidenciaram efeitos limitados sobre os marcadores somáticos, observando-se resultados controversos em relação ao peso corporal, IMC e percentual de gordura corporal. Enquanto Tabor ${ }^{20}$ reportaram efeitos benéficos sobre o peso corporal, Faude e colaboradores ${ }^{14}$ e Hansen e colegas $^{21}$ não observaram diferenças significativas. O mesmo pôde ser observado para o IMC, tendo Rego e Seabra ${ }^{22}$, Weintraub et al. ${ }^{15}$ e Vasconcellos e colegas ${ }^{23}$ descrito efeitos benéficos advindos dos PFR, contrariando os resultados de Seabra et al..$^{25}$, Madsen et al. ${ }^{19}$ e Leach e Yates ${ }^{24}$. Para o percentual de gordura corporal temos o mesmo panorama, com associações positivas em relação aos PFR encontradas por Rego e Seabra ${ }^{22}$ e Vasconcellos e colegas ${ }^{23}$, mas não por Seabra et al. $^{25}$. Vasques e colegas ${ }^{26}$ concluíram em sua meta-análise que os programas que se concentravam na AF e reeducação alimentar das crianças e adolescentes foram os mais bem sucedidos na redução do IMC.

Variáveis como o percentual de gordura intra-abdominal ${ }^{22}$, razão cintura/altura $^{22}$, circunferência da cintura ${ }^{23}$, densidade mineral óssea ${ }^{22}$ e massa muscular ${ }^{22}$, apesar de apresentarem relações positivas com os PFR, foram analisadas por um número limitado de estudos, inviabilizando qualquer generalização ou comparação de seus resultados.

Dos nove estudos elegidos, Hansen e colegas ${ }^{21}$, Rego e Seabra ${ }^{22}$ e Vasconcellos e colegas ${ }^{23}$ encontraram efeitos positivos da aplicação de PFR sobre os marcadores bioquímicos em crianças e adolescentes obesos, observando diminuições nos valores da proteína C-reativa, resistência à insulina, colesterol total, colesterol LDL e triglicerídeos, tal como observado um aumento do colesterol HDL. Já os marcadores inflamatórios leptina, resistina e adiponectina foram analisados somente por Rego e Seabra ${ }^{22}$, que reportou melhoras significativas nos valores de leptina e resistina, além de um aumento significativo da adiponectina. Apesar de limitados, a originalidade destes resultados destaca e reforça o potencial da prática do futebol recreativo na prevenção da obesidade de crianças e adolescentes.

Variáveis cardiovasculares como a pressão arterial (sistólica ou diastólica), frequência cardíaca de repouso, atividade simpática e parassimpática e a estrutura e função cardíaca foram observadas nos trabalhos de Hansen e colegas ${ }^{21}$, Rego e Seabra $^{22}$ e Vasconcellos e colegas ${ }^{23}$. Seus resultados indicaram uma diminuição da pressão arterial e frequência cardíaca de repouso, uma diminuição da atividade simpática e um aumento significativo da atividade parassimpática, assim como efeitos consideráveis sobre o sistema cardiovascular, incluindo a modulação da estrutura e da função cardíaca como consequência da aplicação de PFR.

Dentre as escalas psicossociais estudadas, Faude e colaboradores ${ }^{14}$ só evidenciou resultado positivo para a escala de autoestima ao aplicar o Questionário de Qualidade de vida para Crianças. Seus resultados são condizentes aos encontrados por Seabra et $\mathrm{al}^{25}$ e Rego e Seabra ${ }^{22}$ quanto à autoestima, que também revelaram melhorias nas escalas de imagem corporal, atração em participar em atividade física e competência física percebida após o programa de futebol recreativo.

A baixa magnitude dos resultados evidenciados nesta revisão pode ser explicada pela grande diversidade metodológica aplicada pelos diferentes estudos. Não obstante, é importante notar que somente um dos estudos forneceu informações sobre a idade biológica das crianças aleatorizadas ${ }^{23}$. Questões relacionadas com a matu- 
ração sexual também podem afetar estes resultados de forma enganosa, portanto, futuras pesquisas devem considerar a classificação de maturidade sexual como covariável para verificar o tamanho do efeito dos programas de intervenção ${ }^{27}$.

É de grande interesse para este campo de estudo que futuras investigações pudessem realizar pesquisas de larga-escala, preferencialmente ensaios aleatórios randomizados, que utilizem programas de futebol recreativo em "doses" de treinamento variadas (na sua intensidade, duração e frequência), a fim de se confirmar e aprofundar os resultados aqui apresentados. A identificação de relações entre fatores de risco específicos a diferentes programas de futebol recreativo é também de grande valia para a área, no que sugerimos que estudos futuros procurem definir os critérios de inclusão de seus participantes não exclusivamente orientados pelo IMC ou alguma componente da composição corporal, mas antes por algum fator de risco específico ou agregação (cluster) de fatores de risco.

Concluímos, portanto, que apesar de existirem evidências dos efeitos positivos do futebol recreativo sobre o controle das comorbidades associadas à obesidade, sobretudo nos marcadores bioquímicos e inflamatórios, cardiovasculares e psicossociais, este campo de estudo ainda necessita de mais pesquisas para um melhor aprofundamento e conhecimento sobre a temática em questão.

Apesar das limitações e dificuldades de comparação entre os estudos, é possível considerar que a prática de futebol recreativo como uma forma eficaz, acessível, mas particularmente "não farmacológica", de prevenção/redução deste grave problema de saúde pública, cujas consequências a médio longo prazo ainda nos são impossíveis de imaginar. Destaca-se com os resultados desta revisão sistemática a necessidade de mais pesquisas no contexto de replicação e a "tradução" potencial mais pronunciada em estudos a longo prazo e em larga escala.

\section{Contribuição dos autores}

Os autores Augusto Pedretti e Alessandro Pedretti foram responsáveis por todo o processo de Identificação, Triagem, Elegibilidade e Inclusão dos estudos sob a supervisão do autor André Seabra. Sempre que houve qualquer dúvida durante este processo o autor André Seabra era acionado para decisão final.

Os autores Augusto e Alessandro Pedretti também foram responsáveis por redigir todo o manuscrito de forma co-participativa (resumo, introdução, metodologia, resultados e discussão) sob a supervisão do autor André Seabra que atuou de forma significativa no direcionamento estrutural do manuscrito, polimento e redação quando se julgou necessário.

Durante o processo de revisão pela RBAFS o autor Augusto Pedretti ficou responsável pela retificação e envio das sugestões, que eram realizadas inicialmente em conjunto pelos autores Augusto, Alessandro e André e executada pelo autor Augusto.

\section{Referências}

1. Daniels SR, Arnett DK, Eckel RH, Gidding SS, Hayman LL, Kumanyika S, et al. Overweight in children and adolescents pathophysiology, consequences, prevention, and treatment. Circulation. 2005;111(15):1999-2012.

2. Daniels SR, Jacobson MS, McCrindle BW, Eckel RH, Sanner BM. American Heart Association Childhood Obesity Research Summit Executive Summary. Circulation. 2009;119(15):2114-23.

3. Ebbeling CB, Pawlak DB, Ludwig DS. Childhood obesity: public-health crisis, common sense cure. Lancet. 2002;360(9331):473-82.

4. Han JC, Lawlor DA, Kimm S. Childhood obesity. The Lancet. 2010;375(9727):1737-48.

5. Nadeau KJ, Maahs DM, Daniels SR, Eckel RH. Childhood obesity and cardiovascular disease: links and prevention strategies. Nat Rev Cardiol. 2011;8(9):513-25. 
6. Andersen LB, Harro M, Sardinha LB, Froberg K, Ekelund U, Brage S, et al. Physical activity and clustered cardiovascular risk in children: a cross-sectional study (The European Youth Heart Study). Lancet. 2006;368(9532):299-304.

7. IBGE. Ministério do Planejamento, Orçamento e Gestão. Pesquisa sobre Padrões de Vida 2008-2009. $2^{\text {a }}$ ed. Rio de Janeiro: IBGE. 2010.

8. Semenick D. TESTS AND MEASUREMENTS: The T-test. Strength Cond J. 1990;12(1):36-7.

9. Pereira J, Mateus C. Custos indirectos associados à obesidade em Portugal. Rev Port Sau Pub. 2003;3:65-80.

10. Harris KC, Kuramoto LK, Schulzer M, Retallack JE. Effect of school-based physical activity interventions on body mass index in children: a meta-analysis. Can Med Assoc J. 2009;180(7):719-26.

11. Lavelle H, Mackay D, Pell J. Systematic review and meta-analysis of school-based interventions to reduce body mass index. J Public Health. 2012;34(3):360-9.

12. Meyer AA, Kundt G, Lenschow U, Schuff-Werner P, Kienast W. Improvement of early vascular changes and cardiovascular risk factors in obese children after a six-month exercise program. J Am Coll Cardiol. 2006;48(9):1865-70.

13. Parfitt G, Eston RG. The relationship between children's habitual activity level and psychological well-being. Acta Paediatr. 2005;94(12):1791-7.

14. Faude O, Kerper O, Multhaupt M, Winter C, Beziel K, Junge A, et al. Football to tackle overweight in children. Scand J Med Sci Spor. 2010;20(s1):103-10.

15. Weintraub DL, Tirumalai EC, Haydel KF, Fujimoto M, Fulton JE, Robinson TN. Team Sports for Overweight Children: The Stanford Sports to Prevent Obesity Randomized Trial (SPORT). Arch Pediatr Adolesc Med. 2008;162(3):232-7.

16. Armstrong N, Bray S. Physical activity patterns defined by continuous heart rate monitoring. Arch Dis Child. 1991;66(2):245-7.

17. Randers MB, Nybo L, Petersen J, Nielsen JJ, Christiansen L, Bendiksen M, et al. Activity profile and physiological response to football training for untrained males and females, elderly and youngsters: influence of the number of players. Scand J Med Sci Spor. 2010;20(s1):14-23.

18. Organization WH. Global recommendations on physical activity for health. WHO Library Cataloguing-in-Publication Data; Disponível: http://wwwwhoint/dietphysicalactivity/ publications/9789241599979/en/. 2010.

19. Madsen K, Thompson H, Adkins A, Crawford Y. School-community partnerships: A clusterrandomized trial of an after-school soccer program. Jama Pediatr. 2013;167(4):321-6.

20. Tabor FC. An Examination of the Effects of Type of Sport Participation on Weight Classification and Academic Achievement: Academic Persistence as a Predictor. DePaul Discoveries. 2014;3(1):16.

21. Hansen PR, Andersen LJ, Rebelo AN, Brito J, Hornstrup T, Schmidt JF, et al. Cardiovascular effects of 3 months of football training in overweight children examined by comprehensive echocardiography: a pilot study. J Sports Sci. 2013;31(13):1432-40.

22. Rêgo C, Seabra A. O futebol recreativo como meio não farmacológico no combate à obesidade pediátrica: um projeto patrocinado pela UEFA. Rev Medicina Desportiva informa. 2014;5(4):18-20.

23. Vasconcellos F, Seabra A, Cunha F, Montenegro R, Penha J, Bouskela E, et al. Health markers in obese adolescents improved by a 12 -week recreational soccer program: a randomised controlled trial. J Sports Sci. 2015(ahead-of-print):1-12.

24. Leach RA, Yates JM. Nutrition and youth soccer for childhood overweight: a pilot novel chiropractic health education intervention. J Manipulative Physiol Ther. 2008;31(6):434-41.

25. Seabra A, Seabra A, Brito J, Krustrup P, Hansen PR, Mota J, et al. Effects of a 5-month football program on perceived psychological status and body composition of overweight boys. Scand J Med Sci Spor. 2014;24(S1):10-6.

26. Vasques C, Magalhães P, Cortinhas A, Mota P, Leitão J, Lopes VP. Effects of intervention programs on child and adolescent BMI: A meta-analysis study. J Phys Act Health. 2014;11(2):426-44.

27. Kain J, Uauy R, Vio F, Cerda R, Leyton B. School-based obesity prevention in Chilean primary school children: methodology and evaluation of a controlled study. Int J Obes. 2004;28(4):483-93.

ENDEREÇO PARA CORRESPONDÊNCIA ANDRÉ SEABRA aseabra@fade.up.pt
Faculdade de Desporto, Universidade

do Porto, Portugal,

Rua Dr. Plácido Costa, 91

4200-450 Porto, Portugal. 